\title{
Some Existence and Uniqueness Results for a Time-Dependent Coupled Problem of the Navier-Stokes kind
}

\author{
B. Climent and E. Fernández-CarA*
}

\begin{abstract}
In this paper, we consider some systems which are close to the instationary Navier-Stokes equations. The structure of these systems is the following: An $(N+1)$-dimensional equation for motion (including the incompressibility condition) and a scalar equation involving an additional unknown, $k=k(x, t)$. Among other things, they serve to model the behavior of certain turbulent flows. We are mainly concerned with existence and uniqueness results. The main difficulties are due to the scalar equation. In particular, the right side is typically in $L^{1}$; furthermore, there are nonlinear terms of the kind $\nabla \cdot(\mu(k) \nabla k)$ and $\nabla \cdot(B(k))$, where $\mu$ and $B$ are general continuous functions (no growth condition at infinity is imposed). Following the previous work of other authors, it is crucial to introduce the notion of weak-renormalized solution. Our results provide existence in the two-dimensional case, as well as the uniqueness of regular solution in both the two and three-dimensional cases.
\end{abstract}

${ }^{*}$ Department of Differential Equations and Numerical Analysis, University of Sevilla, Tarfia s/n, E-41012 Sevilla, Spain. Partially supported by D.G.I.C.Y.T. (Spain), Proyecto PB920696. 


\section{Notation:}

- $L^{1}=L^{1}(\Omega), \quad H_{0}^{1}=H_{0}^{1}(\Omega)$, etc.; $|\cdot|($ resp. $\|\cdot\|)$ denotes the usual norm in $L^{2}$ (resp. $\left.H_{0}^{1}\right)$.

- $H^{-1}=H^{-1}(\Omega)$ is the dual space of $H_{0}^{1} ; \quad\|\cdot\|_{*}$ denotes the usual norm in $H^{-1}$.

- In general, if $X=X(\Omega)$ is a space of functions defined in the open set $\Omega$ and $p \geq 1$, we denote by $L^{p}(X)$ (resp. $\left.C^{0}(X)\right)$ the Banach space $L^{p}(0, T ; X)\left(\operatorname{resp} . C^{0}([0, T] ; X)\right)$.

- $z_{+}=\max (z, 0)$ for any real $z$. Contrarily, $z^{+}$(resp. $z^{-}$) denotes any real number $z^{\prime}>z$ (resp. $z^{\prime}<z$ ) close enough to $z$.

- $T_{M}(s)=s$ if $s \in[-M, M] ; T_{M}(s)=M \operatorname{sign} s$ otherwise.

- $\xi_{n, m}=\frac{1}{m}\left(T_{n+m}-T_{n}\right)$ for all $n, m \geq 1$.

- $S_{\delta}(s)=s$ if $s \in[-\delta, \delta] ; S_{\delta}(s)=\operatorname{sign} s$ otherwise.

- $S: D=\sum_{i, j=1}^{N} S_{i j} D_{i j}$ for any $S=\left\{S_{i j}\right\}$ and $D=\left\{D_{i j}\right\}$.

- For each $p \in[1,+\infty], p^{*}$ is the associated Sobolev embedding exponent: $p^{*}=\frac{N p}{N-p}$ if $p<N ; 1<p^{*}<+\infty$ (arbitrary) if $p=N$ and $p^{*}=+\infty$ otherwise. 


\section{Introduction. Motivation of the problem}

This paper is concerned with some nonlinear partial differential systems stemming from fluid mechanics. These are variants of the instationary Navier-Stokes equations and read as follows:

$$
\left\{\begin{aligned}
\partial_{t} u-\nabla \cdot\left(\nu D u+|k|^{1 / 2} \Phi^{\prime}(D u)\right)+(u \cdot \nabla) u+\nabla p=f, \\
\nabla \cdot u=0, \\
\partial_{t} k-\nabla \cdot(\mu(k) \nabla k+B(k))+u \cdot \nabla k=\nu^{\prime}|D u|^{2}+|k|^{1 / 2} \Phi^{\prime}(D u): D u \\
-|k|^{3 / 2} \psi^{0}(D u) .
\end{aligned}\right.
$$

In (1), it is assumed that $D u=\nabla u+{ }^{t} \nabla u$. The functions $D \mapsto \Phi(D)$, $D \mapsto \psi^{0}(D), k \mapsto \mu(k)$ and $k \mapsto B(k)$ are prescribed. Given an open set $\Omega \subset \mathbb{R}^{N}$, a final time $T>0$, the function $f$ and the coefficients $\nu>0$ and $\nu^{\prime} \in[0, \nu]$, we will search for a triplet $\{u, p, k\}$ satisfying (1) in $Q=\Omega \times(0, T)$, together with appropriate initial conditions at $t=0$ and boundary conditions on $\partial \Omega \times(0, T)$.

Systems like (1) are motivated by turbulence modelling. More precisely, let $U=U(x, t)$ and $P=P(x, t)$ be respectively the velocity field and pressure distribution of the turbulent flow of a viscous incompressible fluid. Then, the couple $\{U, P\}$ must satisfy the instationary Navier-Stokes equations:

$$
\partial_{t} U-\nu \Delta U+(U \cdot \nabla) U+\nabla P=F, \quad \nabla \cdot U=0 .
$$

Let us denote by $u$ and $p$ some averaged variables (we write $u=\bar{U}$ and $p=\bar{P}$; $u$ and $p$ describe the mean flow). Let us put

$$
U=u+u^{\prime}, \quad P=p+p^{\prime} .
$$

Then, instead of (2), it is appropriate to try to solve a system that should be satisfied by $u$ and $p$. After some computations, one finds:

$$
\partial_{t} u-\nabla \cdot(\nu D u+R)+(u \cdot \nabla) u+\nabla p=f, \quad \nabla \cdot u=0,
$$

where $f=f(x, t)$ is the averaged external forces field, i.e. $f=\bar{F}$ and $R$ is the so called Reynolds tensor:

$$
R=\left\{R_{i j}\right\}, \quad \text { with } R_{i j}=-\overline{u_{i}^{\prime} u_{j}^{\prime}} .
$$

Since in (3) we still find the unknown variables $u_{i}^{\prime}$, it is reasonable to introduce closing hypotheses relating $R$ to $u$. In the case of usual one-equation models, one imposes the following hypothesis of the Boussinesq kind:

$$
R=\nu_{T} D u, \quad \text { where } \nu_{T}=\nu_{T}(k) \quad \text { (an algebraic relation) }
$$

Here, $k=\overline{\frac{1}{2}\left|u^{\prime}\right|^{2}}$ is the mean turbulent kinetic energy. The problem is thus closed using (3), (4) and an additional PDE for $k$. 
Unfortunately, when one tries to deduce an equation for $k$, one finds again terms in which the turbulent perturbations $u_{i}^{\prime}$ (and $k^{\prime}$ ) appear. More precisely, one has:

$$
\partial_{t} k-\nabla \cdot\left(\nu \nabla k+\left(\overline{\left(-\left(p^{\prime}+k^{\prime}\right) u^{\prime}\right.}\right)\right)+u \cdot \nabla k=R: D u-\overline{\frac{\nu}{2}\left|D u^{\prime}\right|^{2}} .
$$

Consequently, one has to replace (5) by an approximation. This is made by introducing new closing hypotheses:

- Of course, (4) is used again in order to approximate the production term $R: D u$.

- The approximation of the dissipation term $\overline{\frac{\nu}{2}\left|D u^{\prime}\right|^{2}}$ is almost always the same: a constant times $k^{3 / 2}$.

- Contrarily, the approximation of $\overline{-\left(p^{\prime}+k^{\prime}\right) u^{\prime}}$ has been achieved in several different ways in the litterature. In most papers, this term is replaced by $c \nu_{T} \nabla k$, where $c$ is an experimental constant (for instance, see [14], [15] and the references therein). In other papers, however, it is replaced by a vector function $B(k)$ (see [6]).

Hence, it is clear that equations like (1) can be used to describe the evolution of some turbulent flows. Another motivation for (1) can be found in non Newtonian mechanics. In this setting, $u$ and $p$ are the true velocity field and pressure, $k$ is the temperature and it is assumed that the stress tensor $\tau$ depends on $D u$ and $k$ as follows:

$$
\tau=\nu^{\prime} D u+k \Phi^{\prime}(D u)
$$

\section{The main results}

In this section, we present our main results. These are concerned with existence and uniqueness for systems of the kind (1) completed with appropriate initial and boundary conditions. Let us mention that the authors have considered a similar stationary problem in the previous paper [8]. At first sight, one could think that, for (1), the results can be obtained in the same way as in [8]. This is not true at least because of three reasons:

- As shown below, there is a major difficulty for the existence proof when $N=3$. This is related to the lack of regularity of $\partial_{t} u$, typical of the evolution Navier-Stokes problem and its variants.

- In order to prove that $k$ is a solution to the third equation in (1), the arguments used in the stationary case, which have been taken in part from [12], do not work. This is maybe surprising but well known. Here, another argument, essentially due to D. Blanchard and H. Redwane [3], is used. 
- The uniqueness results presented in [8] (and also their proofs) are different from those in this paper. Roughly speaking, in [8] we prove uniqueness for regular data and sufficiently small Reynolds number, i.e. large $\nu$. Of course, it is not reasonable to expect results of this kind in the framework of the time-dependent problem (1). In this paper, we prove that regular solutions, in case they exist, must be unique.

We will consider a simplified version of (1):

$$
\left\{\begin{array}{l}
\partial_{t} u-\nu \Delta u-\nabla \cdot\left(k \Phi^{\prime}(\nabla u)\right)+(u \cdot \nabla) u+\nabla p=f, \\
\nabla \cdot u=0, \\
\partial_{t} k-\nabla \cdot(\mu(k) \nabla k+B(k))+u \cdot \nabla k=\nu|\nabla u|^{2}+k \Phi^{\prime}(\nabla u): \nabla u .
\end{array}\right.
$$

This is made for simplicity; in fact, the results in this section also hold for (1) with appropriate minor changes. The first, second and third equations in (6) will be known as the motion equation, the incompressibility condition and the energy equation, respectively. Our basic assumptions are the following:

- $\Omega \subset \mathbb{R}^{N}$ is a bounded, connected, open and regular set; $T>0, \nu>0$ and $f \in L^{2}\left(H^{-1}\right)$.

- $D \mapsto \Phi(D)$ is $C^{1}, \Phi^{\prime}(0)=0,\left|\Phi^{\prime}(D)\right| \leq$ Const. and $D \mapsto \Phi^{\prime}(D)$ : $D$ is convex (consequently, it is also locally Lipschitz-continuous). In particular, $D \mapsto \Phi(D)$ is convex and one has $\left(\Phi^{\prime}\left(D_{1}\right)-\Phi^{\prime}\left(D_{2}\right)\right):\left(D_{1}-D_{2}\right) \geq 0$ for all $D_{1}$ and $D_{2}$.

- $k \mapsto \mu(k)$ and $k \mapsto B(k)$ are continuous functions; furthermore, $\mu(k) \geq$ $\mu_{0}>0$ for all $k$.

We want to solve (6) in $Q=\Omega \times(0, T)$ together with initial conditions

$$
\left.u\right|_{t=0}=u_{0} \quad \text { and }\left.\quad k\right|_{t=0}=k_{0} \quad \text { in } \quad \Omega
$$

and homogeneous Dirichlet conditions

$$
u=0 \quad \text { and } \quad k=0 \quad \text { on } \quad \partial \Omega \times(0, T) .
$$

Our main interest concerns general continuous functions $\mu$ and $B$. This is motivated by the fact that, in turbulence modelling, an equation exactly satisfied by the true turbulent kinetic energy is unknown. Besides the usual spaces $L^{p}\left(L^{q}\right), L^{2}(V)$, etc., we will use the following:

$$
\begin{gathered}
\mathcal{L}=\left\{\psi \in L^{1}(Q) ; T_{M}(\psi) \in L^{2}\left(H_{0}^{1}\right) \quad \forall M>0,\right. \\
\left.\lim _{n \rightarrow+\infty} \frac{1}{n} \iint_{n \leq|\psi| \leq 2 n}|\nabla \psi|^{2} d x d t=0\right\}
\end{gathered}
$$


(see the Notation). Moreover, $\widetilde{\beta}, \widetilde{\mu}$, etc. will denote integrals of the corresponding $\beta, \mu, \ldots$ For instance,

$$
\widetilde{\beta}(s)=\int_{0}^{s} \beta(\sigma) d \sigma \quad \text { for all } s .
$$

TheOREm 1 - Assume $N=2, u_{0} \in V$ and $k_{0} \in L^{1}$, with $k_{0} \geq 0$. Under the previous assumptions, there exists $\{u, p, k\}$, with

$$
u \in L^{2}(V) \cap C^{0}(H), \quad p \in L^{2}(Q), \quad k \in \mathcal{L},
$$

such that:

1. The couple $\{u, p\}$ solves the first two equations in (6) together with the first initial condition in (7) in the usual weak sense.

2. $k \geq 0$ and solves the energy equation in (6) and the second initial condition in $(7)$ in the following sense: For all $\beta \in W^{1, \infty}(\mathbb{R})$ with compact support, one has

$$
\left\{\begin{array}{l}
\partial_{t} \widetilde{\beta}(k)-\nabla \cdot(\beta(k)(\mu(k) \nabla k+B(k))) \\
\quad+\beta^{\prime}(k) \nabla k \cdot(\mu(k) \nabla k+B(k))+\beta(k)(u \cdot \nabla k) \\
\quad=\beta(k)\left(\nu|\nabla u|^{2}+k \Phi^{\prime}(\nabla u): \nabla u\right) \quad \text { in } \mathcal{D}^{\prime}(Q) .
\end{array}\right.
$$

In particular, $\partial_{t} \widetilde{\beta}(k) \in L^{1}\left(L^{1}\right)+L^{2}\left(H^{-1}\right)$ and, for all $q<2$, one has $\widetilde{\beta}(k) \in C^{0}\left(W^{-1, q}\right)$. Furthermore,

$$
\left.\widetilde{\beta}(k)\right|_{t=0}=\widetilde{\beta}\left(k_{0}\right) .
$$

A triplet $\{u, p, k\}$ as above will be called a weak-renormalized solution to (6), (7), (8). Renormalized solutions to PDE's have been introduced by R. DiPerna and P.L. Lions in [9], in the framework of the Boltzmann equations. They have been used in connection with various nonlinear elliptic (resp. parabolic) equations by P. Benilan et al. [2], L. Boccardo et al. [5] and P.L. Lions and F. Murat [12],[13] (resp. by D. Blanchard and H. Redwane [3]). For the analysis of some problems similar to (1) and (6), weak-renormalized solutions were first considered by R. Lewandowski [11]. That we search for a renormalized solution $k$ is motivated by the structure of the right side of the energy equation in (6) (typically in $L^{1}$ ) and also by our interest in keeping $\mu$ and $B$ as general as possible. 
THEOREM 2 - Under the assumptions in theorem 1 , assume also that $B \equiv 0$ and $k_{0} \in L^{\infty}$. Then the solution $\{u, p, k\}$ furnished by theorem 1 satisfies

$$
\begin{aligned}
& \widetilde{\mu}(k) \in \bigcap_{q<2} L^{q}\left(W_{0}^{1, q}\right), \quad \nabla \widetilde{\mu}(k)=\mu(k) \nabla k, \\
& \partial_{t} k \in L^{1}\left(L^{1}\right)+L^{q}\left(W^{-1, q}\right) \quad \text { for all } q<2
\end{aligned}
$$

and also the following:

$$
\left\{\begin{array}{l}
\left\langle\partial_{t} k, \phi\right\rangle+\int_{\Omega} \mu(k) \nabla k \cdot \nabla \phi+\int_{\Omega}(u \cdot \nabla k) \phi \\
=\int_{\Omega}\left(\nu|\nabla u|^{2}+k \Phi^{\prime}(\nabla u): \nabla u\right) \phi \quad \forall \phi \in \mathcal{D}(\Omega), \quad \text { a.e. in }(0, T) .
\end{array}\right.
$$

If $\{u, p, k\}$ is as in theorem 2 , it will be said it is a weak solution to (6), (7), (8). As mentioned above, the situation considered in this theorem is the most frequently found in connection with one-equation turbulence models.

Theorem 3 -Assume that $N=2$ or $N=3, k \mapsto \mu(k)$ is locally Lipschitzcontinuous, $B \equiv 0$ and

$$
D \mapsto \Phi(D) \quad \text { is } C^{2} \text {, with }\left|\Phi^{\prime \prime}(D)\right| \leq \text { Const. }
$$

Let $u_{0} \in V$ and $k_{0} \in W^{2, \infty} \cap H_{0}^{1}$, with $k_{0} \geq 0$. For $i=1,2$, let $\left\{u_{i}, p_{i}, k_{i}\right\}$ be a (weak) solution to (6), (7), (8), with $u_{i} \in L^{\infty}\left(W^{1, \infty}\right)$ and suppose that (for instance) $u_{2} \in L^{2}\left(W^{2, r}\right)$, where $r>N(r \geq 3$ if $N=3)$. Then $\left\{u_{1}, \nabla p_{1}, k_{1}\right\}$ and $\left\{u_{2}, \nabla p_{2}, k_{2}\right\}$ must coincide.

Before giving the proofs of these results, let us make some remarks:

1. Theorems 1 and 2 provide existence for (6), together with suitable initial and boundary conditions when $N=2$. Other similar results can be found in [11]. It is not clear how to extend the proofs in order to cover the three-dimensional case (see the second and subsequent steps of the proof of theorem 1).

2. However, several more or less obvious generalizations of theorems 1 and 2 are possible. Thus, an existence result similar to theorem 1 can be deduced if we replace $k \Phi^{\prime}(\nabla u)$ by a term of the form $D_{2} \Psi(k, \nabla u)$, where $(s, D) \mapsto \Psi(s, D)$ satisfies appropriate assumptions. When $N=3$, existence can also be obtained if one omits the nonlinear term $(u \cdot \nabla) u$. On 
the other hand, theorem 2 holds as well when $B$ is not zero but Lipschitzcontinuous; for details, see [7]. An interesting situation arises when we simply assume $\mu(k) \geq 0$ in (6). It seems also interesting to relax the assumption " $D \mapsto \Phi^{\prime}(D): D$ is convex" in order to account for more general approximations of the Reynolds tensor. These cases are far from trivial and will be considered in a future work.

3. Some variants of theorem 3 can also be proved. Nevertheless, there is an important question that remains open: For $N=2$, is there uniqueness of weak-renormalized solution? This seems to be complicate, but a positive answer would have a very interesting interpretation.

4. The existence of a weak-renormalized solution (and the existence and uniqueness of a weak solution) to the stationary analog of (6) have been established by the authors in [8] under reasonable assumptions (see also [1] and [11] for other similar results).

\section{The proof of theorem 1}

In the sequel, $C$ denotes a constant which may depend on $\Omega$ and the other data in (6). The proof of theorem 1 consists of six steps:

FIRST STEP: The introduction of a family of approximations.

For each $\varepsilon>0$, we consider the following approximation to (6):

$$
\left\{\begin{array}{l}
\partial_{t} u^{\varepsilon}-\nabla \cdot \tau^{\varepsilon}+\left(u^{\varepsilon} \cdot \nabla\right) u^{\varepsilon}+\nabla p^{\varepsilon}=f, \\
\nabla \cdot u^{\varepsilon}=0, \\
\partial_{t} k^{\varepsilon}-\nabla \cdot\left(\mu_{\varepsilon}\left(k^{\varepsilon}\right) \nabla k^{\varepsilon}+B_{\varepsilon}\left(k^{\varepsilon}\right)\right)+u^{\varepsilon} \cdot \nabla k^{\varepsilon}=T_{\frac{1}{\varepsilon}}\left(\tau^{\varepsilon}: \nabla u^{\varepsilon}\right) .
\end{array}\right.
$$

Here, we have used the following notation:

$$
\mu_{\varepsilon}=T_{\frac{1}{\varepsilon}} \circ \mu, \quad B_{\varepsilon}=B \circ T_{\frac{1}{\varepsilon}}, \quad \tau^{\varepsilon}=\nu \nabla u^{\varepsilon}+T_{\frac{1}{\varepsilon}}\left(k^{\varepsilon}\right)_{+} \Phi^{\prime}\left(\nabla u^{\varepsilon}\right) .
$$

Again, these equations are required to be satisfied in $Q=\Omega \times(0, T)$ together with the initial conditions

$$
\left.u^{\varepsilon}\right|_{t=0}=u_{0} \quad \text { and }\left.\quad k^{\varepsilon}\right|_{t=0}=T_{\frac{1}{\varepsilon}}\left(k_{0}\right) \quad \text { in } \quad \Omega
$$

and the boundary conditions

$$
u^{\varepsilon}=0 \quad \text { and } \quad k^{\varepsilon}=0 \quad \text { on } \quad \partial \Omega \times(0, T) .
$$

The existence of a triplet $\left\{u^{\varepsilon}, p^{\varepsilon}, k^{\varepsilon}\right\}$ satisfying (13), (14) and (15) can be established using (for instance) a Galerkin method. In fact, with this technique one 
finds some nontrivial difficulties that can be solved arguing as in the following steps. One sees that

$$
u^{\varepsilon} \in L^{2}(V) \cap C^{0}(H), \quad k^{\varepsilon} \in L^{2}\left(H_{0}^{1}\right) \cap C^{0}\left(L^{2}\right)
$$

and, also, that $k^{\varepsilon} \geq 0$.

SECOND STEP: A priori estimates and weak convergence.

Using $u^{\varepsilon}$ as a test function in the first equation in (13), one obtains:

$$
\left|u^{\varepsilon}(t)\right|^{2}+\int_{0}^{t} \int_{\Omega} \tau^{\varepsilon}: \nabla u^{\varepsilon} \leq C \quad \text { in } \quad(0, T) .
$$

In particular,

$$
\left\|u^{\varepsilon}\right\|_{L^{\infty}(H)} \leq C, \quad\left\|u^{\varepsilon}\right\|_{L^{2}(V)} \leq C .
$$

¿From this, one also deduces by interpolation:

$$
u^{\varepsilon} \text { is bounded in } L^{\left(\frac{2 a}{a-2}\right)^{-}}\left(L^{a}\right), \text { for all finite } a>2
$$

(recall that $z^{-}$is, for each $z \in \mathbb{R}$, an arbitrary real number $z^{\prime}<z$, close enough to $z$ ). Since $N=2$, (16) suffices to deduce a bound for $\partial_{t} u^{\varepsilon}$ in $L^{2}\left(V^{\prime}\right)$ :

$$
\left\|\partial_{t} u^{\varepsilon}\right\|_{L^{2}\left(V^{\prime}\right)} \leq C
$$

Let us now consider the third equation in (13) (the energy equation). We will succesively use $S_{\delta}\left(k^{\varepsilon}\right), T_{M}\left(k^{\varepsilon}\right)$ and $\xi_{n, m}\left(k^{\varepsilon}\right)$ as test functions (see the Notation). We easily find:

$$
\left\|k^{\varepsilon}\right\|_{L^{\infty}\left(L^{1}\right)} \leq C, \quad\left\|T_{M}\left(k^{\varepsilon}\right)\right\|_{L^{2}\left(H_{0}^{1}\right)} \leq C \cdot M
$$

and also

$$
\frac{1}{m} \iint_{n \leq k^{\varepsilon} \leq n+m} \mu_{\varepsilon}\left(k^{\varepsilon}\right)\left|\nabla k^{\varepsilon}\right|^{2} \leq \iint_{k^{\varepsilon} \geq n} T_{\frac{1}{\varepsilon}}\left(\tau^{\varepsilon}: \nabla u^{\varepsilon}\right)+\int_{\Omega} \widetilde{\xi}_{n, m}\left(T_{\frac{1}{\varepsilon}}\left(k_{0}\right)\right)
$$

(recall that $\widetilde{\xi}_{n, m}$ is the primitive of $\xi_{n, m}$ which vanishes at zero). From (18), one sees that

$$
\frac{1}{m} \iint_{n \leq k^{\varepsilon} \leq n+m}\left|\nabla k^{\varepsilon}\right|^{2} \leq C(n, m) .
$$

¿From (17) and (19), arguing as in [4] and [13], one deduces the following:

$$
\left\|k^{\varepsilon}\right\|_{L^{q}\left(W_{0}^{1, q}\right)} \leq C_{q} \quad \text { for all } q<2 .
$$

By interpolation, it is also seen that

$$
k^{\varepsilon} \text { is bounded in } L^{\left(\frac{2 b}{b-1}\right)^{-}}\left(L^{b}\right), \text { for all finite } b>1 .
$$


Furthermore, for any compactly supported function $\beta \in W^{1, \infty}(\mathbb{R})$, one has:

$$
\partial_{t} \widetilde{\beta}\left(k^{\varepsilon}\right) \quad \text { is bounded in } \quad L^{1}\left(L^{1}\right)+L^{2}\left(H^{-1}\right) .
$$

This is found by simply multiplying the energy equation in (13) by $\beta\left(k^{\varepsilon}\right)$. In particular, $\partial_{t} \widetilde{\beta}\left(k^{\varepsilon}\right)$ is also uniformly bounded in $L^{1}\left(W^{-1, q}\right)$ for any $q \in(1,2)$.

The above estimates for $u^{\varepsilon}$ and $k^{\varepsilon}$ lead to the existence of subsequences that converge weakly. Indeed, at least for a subsequence, one must have:

$$
\begin{aligned}
& u^{\varepsilon} \rightarrow u \quad \text { weakly in } L^{2}(V) \text {, strongly in } L^{\left(\frac{2 a}{a-2}\right)^{-}}\left(L^{a}\right) \quad \forall a>2 \text { and a.e., } \\
& \partial_{t} u^{\varepsilon} \rightarrow \partial_{t} u \quad \text { weakly in } L^{2}\left(V^{\prime}\right), \\
& k^{\varepsilon} \rightarrow k \quad \text { weakly in } L^{q}\left(W_{0}^{1, q}\right) \quad \forall q<2, \text { strongly in } L^{\left(\frac{2 b}{b-1}\right)^{-}}\left(L^{b^{-}}\right) \quad \forall b>1 \\
& \quad \text { and a.e., } \\
& T_{M}\left(k^{\varepsilon}\right) \rightarrow T_{M}(k) \quad \text { weakly in } L^{2}\left(H_{0}^{1}\right) \quad \forall M>0 .
\end{aligned}
$$

Here, the fact that $N=2$ has been used (if $N=3$, we would have only $u^{\varepsilon} \rightarrow u$ weakly in $\left.L^{4 / 3}\left(V^{\prime}\right)\right)$. Also, notice that $u \in C^{0}(H)$ and $u(0)=u_{0}$. The previous a.e. and strong convergence of $k^{\varepsilon}$ stems from (17) (which must be satisfied for all $M>0$ ) and the fact that, for any $\beta$ as above, one has (20). Obviously, one has $k \geq 0$.

THIRD STEP: $u$ is, together with some $p$, a solution to the motion equation. In order to take limits in the first two equations in (13), it will be convenient to introduce an equivalent variational inequality. For each $\varepsilon>0, u^{\varepsilon}$ is a solution to the following:

$$
\left\{\begin{array}{l}
\int_{0}^{T}\left\langle\partial_{t} u^{\varepsilon}, v-u^{\varepsilon}\right\rangle+\nu \iint_{Q} \nabla u^{\varepsilon}:\left(\nabla v-\nabla u^{\varepsilon}\right) \\
\quad+\iint_{Q}\left(u^{\varepsilon} \cdot \nabla\right) u^{\varepsilon} \cdot\left(v-u^{\varepsilon}\right)+\iint_{Q} T_{\frac{1}{\varepsilon}}\left(k^{\varepsilon}\right) \Phi(\nabla v)-\iint_{Q} T_{\frac{1}{\varepsilon}}\left(k^{\varepsilon}\right) \Phi\left(\nabla u^{\varepsilon}\right) \\
\quad \geq \int_{0}^{T}\left\langle f, v-u^{\varepsilon}\right\rangle \quad \forall v \in V, \quad u^{\varepsilon} \in V .
\end{array}\right.
$$

This can also be written as follows:

$$
\left\{\begin{array}{c}
\int_{0}^{T}\left\langle\partial_{t} u^{\varepsilon}, v\right\rangle+\nu \iint_{Q} \nabla u^{\varepsilon}: \nabla v+\iint_{Q}\left(u^{\varepsilon} \cdot \nabla\right) u^{\varepsilon} \cdot v+\iint_{Q} T_{\frac{1}{\varepsilon}}\left(k^{\varepsilon}\right) \Phi(\nabla v) \\
+\frac{1}{2}\left|u_{0}\right|^{2} \geq \nu \iint_{Q}\left|\nabla u^{\varepsilon}\right|^{2}+\iint_{Q} T_{\frac{1}{\varepsilon}}\left(k^{\varepsilon}\right) \Phi\left(\nabla u^{\varepsilon}\right) \\
+\int_{0}^{T}\left\langle f, v-u^{\varepsilon}\right\rangle+\frac{1}{2}\left|u^{\varepsilon}(T)\right|^{2} \quad \forall v \in V, \quad u^{\varepsilon} \in V .
\end{array}\right.
$$


Taking limits as $\varepsilon \rightarrow 0$, one obtains:

$$
\begin{aligned}
& \int_{0}^{T}\left\langle\partial_{t} u, v\right\rangle+\nu \iint_{Q} \nabla u: \nabla v+\iint(u \cdot \nabla) u \cdot v+\iint_{Q} k \Phi(\nabla v) \\
& \quad+\frac{1}{2}\left|u_{0}\right|^{2} \geq \nu \liminf _{\varepsilon \rightarrow 0} \iint_{Q}\left|\nabla u^{\varepsilon}\right|^{2}+\liminf _{\varepsilon \rightarrow 0} \iint_{Q} T_{\frac{1}{\varepsilon}}\left(k^{\varepsilon}\right) \Phi\left(\nabla u^{\varepsilon}\right) \\
& \quad+\langle f, v-u\rangle+\frac{1}{2}|u(T)|^{2} .
\end{aligned}
$$

Here, $N=2$ is needed: we have used the weak convergence in $H$ of $u^{\varepsilon}(T)$. The first term in the right is bounded from below by

$$
\nu \iint_{Q}|\nabla u|^{2}
$$

In what concerns the second term, let us notice that

$$
\iint_{Q} T_{\frac{1}{\varepsilon}}\left(k^{\varepsilon}\right) \Phi\left(\nabla u^{\varepsilon}\right)=\iint_{Q}\left(T_{\frac{1}{\varepsilon}}\left(k^{\varepsilon}\right)-k\right) \Phi\left(\nabla u^{\varepsilon}\right)+\iint_{Q} k \Phi\left(\nabla u^{\varepsilon}\right) .
$$

Thus, taking into account that the function

$$
v \mapsto \iint_{Q} k \Phi(\nabla v)
$$

is lower semicontinous, we find:

$$
\begin{gathered}
\liminf _{\varepsilon \rightarrow 0} \iint_{Q} T_{\frac{1}{\varepsilon}}\left(k^{\varepsilon}\right) \Phi\left(\nabla u^{\varepsilon}\right) \geq \lim _{\varepsilon \rightarrow 0} \iint_{Q}\left(T_{\frac{1}{\varepsilon}}\left(k^{\varepsilon}\right)-k\right) \Phi\left(\nabla u^{\varepsilon}\right) \\
+\liminf _{\varepsilon \rightarrow 0} \iint_{Q} k \Phi\left(\nabla u^{\varepsilon}\right) \geq \iint_{Q} k \Phi(\nabla u) .
\end{gathered}
$$

Consequently, $u$ is a solution to the variational inequality

$$
\left\{\begin{array}{l}
\int_{0}^{T}\left\langle\partial_{t} u, v-u\right\rangle+\nu \iint_{Q} \nabla u:(\nabla v-\nabla u) \\
\quad+\iint_{Q}(u \cdot \nabla) u \cdot(v-u)+\iint_{Q} k \Phi(\nabla v)-\iint_{Q} k \Phi(\nabla u) \\
\quad \geq \int_{0}^{T}\langle f, v-u\rangle \quad \forall v \in V, \quad u \in V .
\end{array}\right.
$$

Introducing in (21) test functions $v$ of the form $u+\lambda w$, where $w \in V$ and $\lambda \in \mathbb{R}$ and letting $\lambda \rightarrow 0$, it is quite easy to prove that $u$ solves, together with some 
$p \in L^{2}$, the first two equations in (6) in the usual weak sense.

FOURTH STEP: The strong convergence of $\nabla u^{\varepsilon}$.

For almost $t$, let us use $u(t)$ as function in the motion equation in (6). Integrating twice with respect to time, one obtains:

$$
\begin{gathered}
\frac{1}{2} \int_{0}^{T}|u|^{2}+\iint_{Q}(T-t)\left(\nu|\nabla u|^{2}+k \Phi^{\prime}(\nabla u): \nabla u\right) \\
=\int_{0}^{T}(T-t)\langle f, u\rangle+\frac{T}{2}\left|u_{0}\right|^{2} .
\end{gathered}
$$

Here, we have used the fact that $\partial_{t} u \in L^{2}\left(V^{\prime}\right)$, which is true because $N=2$. On the other hand, choosing $u^{\varepsilon}$ as test function in the first equation in (13), one also has:

$$
\begin{gathered}
\frac{1}{2} \int_{0}^{T}\left|u^{\varepsilon}\right|^{2}+\iint_{Q}(T-t)\left(\nu\left|\nabla u^{\varepsilon}\right|^{2}+T_{\frac{1}{\varepsilon}}\left(k^{\varepsilon}\right) \Phi^{\prime}\left(\nabla u^{\varepsilon}\right): \nabla u^{\varepsilon}\right) \\
=\int_{0}^{T}(T-t)\left\langle f, u^{\varepsilon}\right\rangle+\frac{T}{2}\left|u_{0}^{\varepsilon}\right|^{2} .
\end{gathered}
$$

Consequently,

$$
\begin{gathered}
\lim _{\varepsilon \rightarrow 0} \iint_{Q}(T-t)\left(\nu\left|\nabla u^{\varepsilon}\right|^{2}+T_{\frac{1}{\varepsilon}}\left(k^{\varepsilon}\right) \Phi^{\prime}\left(\nabla u^{\varepsilon}\right): \nabla u^{\varepsilon}\right) \\
=\iint_{Q}(T-t)\left(\nu|\nabla u|^{2}+k \Phi^{\prime}(\nabla u): \nabla u\right) .
\end{gathered}
$$

Since $\Phi^{\prime}$ is uniformly bounded, one finds:

$$
\begin{aligned}
& 0=\lim _{\varepsilon \rightarrow 0}\left(\iint_{Q}(T-t)\left(\nu\left|\nabla u^{\varepsilon}\right|^{2}+k \Phi^{\prime}\left(\nabla u^{\varepsilon}\right): \nabla u^{\varepsilon}\right)\right. \\
& \left.-\iint_{Q}(T-t)\left(\nu|\nabla u|^{2}+k \Phi^{\prime}(\nabla u): \nabla u\right)\right) \\
& =\lim _{\varepsilon \rightarrow 0}\left(2 \nu \iint_{Q}(T-t) \nabla\left(u^{\varepsilon}-u\right): \nabla u+\nu \iint_{Q}(T-t)\left|\nabla\left(u^{\varepsilon}-u\right)\right|^{2}\right. \\
& \left.+\iint_{Q}(T-t) k \Phi^{\prime}\left(\nabla u^{\varepsilon}\right): \nabla u^{\varepsilon}-\iint_{Q}(T-t) k \Phi^{\prime}(\nabla u): \nabla u\right) \\
& \geq \limsup _{\varepsilon \rightarrow 0}\left(\nu \iint_{Q}(T-t)\left|\nabla\left(u^{\varepsilon}-u\right)\right|^{2}\right) \\
& +\liminf _{\varepsilon \rightarrow 0}\left(\iint_{Q}(T-t) k \Phi^{\prime}\left(\nabla u^{\varepsilon}\right): \nabla u^{\varepsilon}-\iint_{Q}(T-t) k \Phi^{\prime}(\nabla u): \nabla u\right) .
\end{aligned}
$$


Here, the last term is $\geq 0$, in view of the lower semicontinuity of the function

$$
v \mapsto \iint_{Q}(T-t) k \Phi^{\prime}(\nabla v): \nabla v .
$$

Thus,

$$
\lim _{\varepsilon \rightarrow 0} \iint_{Q}(T-t)\left|\nabla\left(u^{\varepsilon}-u\right)\right|^{2}=0
$$

whence we deduce that

$$
\nabla u^{\varepsilon} \rightarrow \nabla u \quad \text { strongly in } L^{2}\left(\Omega \times\left(0, T^{-}\right)\right) .
$$

FifTH STEP: For all $M>0, T_{M}\left(k^{\varepsilon}\right)$ converges strongly.

First, we write the energy equation in (13) in the form

$$
\partial_{t} k^{\varepsilon}-\nabla \cdot\left(\mu_{\varepsilon}\left(k^{\varepsilon}\right) \nabla k^{\varepsilon}+B_{\varepsilon}\left(k^{\varepsilon}\right)\right)=F_{1}^{\varepsilon}+F_{2}^{\varepsilon},
$$

with

$$
F_{1}^{\varepsilon}=T_{\frac{1}{\varepsilon}}\left(\tau^{\varepsilon}: \nabla u^{\varepsilon}\right), \quad F_{2}^{\varepsilon}=-u^{\varepsilon} \cdot \nabla k^{\varepsilon} .
$$

We already know that

$$
F_{1}^{\varepsilon} \rightarrow \nu|\nabla u|^{2}+k \Phi^{\prime}(\nabla u): \nabla u \text { strongly in } L^{1}\left(\Omega \times\left(0, T^{-}\right)\right) .
$$

On the other hand, $F_{2}^{\varepsilon}$ converges weakly in $L^{c}(Q)$ for some $c>1$. This last assertion can be demonstrated from the convergence properties deduced in the second step as follows. One has:

$$
\nabla k^{\varepsilon} \rightarrow \nabla k \text { weakly in } L^{q}(Q) \text { for all } q<2 .
$$

On the other hand, recall that

$$
u^{\varepsilon} \rightarrow u \text { strongly in } L^{\left(\frac{2 a}{a-2}\right)^{-}}\left(L^{a}\right) \text { for all finite } a>2 .
$$

In particular, if $q<2$ and $a$ is chosen greater than $q^{\prime}$ but close enough, one also has $\frac{2 a}{a-2}>q^{\prime}$, whence it is also true that $u^{\varepsilon} \rightarrow u$ strongly in $L^{p}\left(L^{p}\right)$ for some $p>q^{\prime}$. This, together with (24), gives the desired result.

Now, it can be deduced that

$$
\lim _{\varepsilon \rightarrow 0} \iint_{Q}(T-t)\left|\mu_{\varepsilon}\left(k^{\varepsilon}\right)^{\frac{1}{2}} \nabla T_{M}\left(k^{\varepsilon}\right)-\mu(k)^{\frac{1}{2}} \nabla T_{M}(k)\right|^{2}=0
$$

for each $M>0$. This is due to D. Blanchard and H. Redwane (see [3]). The main consequence is:

$$
\nabla T_{M}\left(k^{\varepsilon}\right) \rightarrow \nabla T_{M}(k) \text { strongly in } L^{2}\left(\Omega \times\left(0, T^{-}\right)\right) .
$$


SiXTH STEP: $k$ is a renormalized solution.

Let us choose $\varphi \in \mathcal{D}(Q)$ and $\beta \in W^{1, \infty}(\mathbb{R})$, with support in $[-M, M]$. Using $\beta\left(k^{\varepsilon}\right) \varphi$ as test function in the energy equation in (22), we find the following:

$$
\begin{aligned}
& \int_{0}^{T}\left\langle\partial_{t} \widetilde{\beta}\left(k^{\varepsilon}\right), \varphi\right\rangle+\iint_{Q} \mu_{\varepsilon}\left(k^{\varepsilon}\right) \nabla k^{\varepsilon} \cdot \nabla\left(\beta\left(k^{\varepsilon}\right) \varphi\right) \\
& \quad+\iint_{Q} B_{\varepsilon}\left(k^{\varepsilon}\right) \cdot \nabla\left(\beta\left(k^{\varepsilon}\right) \varphi\right)=\iint_{Q}\left(F_{1}^{\varepsilon}+F_{2}^{\varepsilon}\right) \beta\left(k^{\varepsilon}\right) \varphi .
\end{aligned}
$$

In these integrals, $k^{\varepsilon}$ can be replaced by $T_{M}\left(k^{\varepsilon}\right)$. Hence, writing $\nabla\left(\beta\left(k^{\varepsilon}\right) \varphi\right)$ in the form $\varphi \nabla \beta\left(k^{\varepsilon}\right)+\beta\left(k^{\varepsilon}\right) \nabla \varphi$ and using the fact that $\nabla T_{M}\left(k^{\varepsilon}\right)$ converges strongly, it is not difficult to take limits as $\varepsilon \rightarrow 0$. One obtains:

$$
\begin{aligned}
\int_{0}^{T}\left\langle\partial_{t} \widetilde{\beta}(k), \varphi\right\rangle & +\iint_{Q}(\mu(k) \nabla k+B(k)) \cdot \nabla(\beta(k) \varphi) \\
= & \iint_{Q}\left(\nu|\nabla u|^{2}+k \Phi^{\prime}(\nabla u): \nabla u-u \cdot \nabla k\right) \beta(k) \varphi .
\end{aligned}
$$

Since $\varphi$ and $\beta$ are arbitrary, this shows that (9) is satisfied.

In order to check that $k$ satisfies the second initial condition in (7) in the sense of (10), we will use the following result, whose proof can be found in [17]:

Lemma- Let $X, B$ and $Y$ be three Banach spaces with $X \subset B \subset Y$, where the first embedding is compact and the second one is continuous. Let $\mathcal{F}$ be a family in $L^{\infty}(X)$ satisfying the following two properties:

1. $\mathcal{F}$ is bounded in $L^{\infty}(X)$.

2. $\partial_{t} v \in L^{1}(Y)$ for each $v \in \mathcal{F}$ and there exist $\psi \in L^{1}(0, T), r>1$ and a bounded set $\mathcal{B}$ in $L^{r}(0, T)$ such that

$$
\left\|\partial_{t} v\right\|_{Y} \in \psi+\mathcal{B} \quad \forall v \in \mathcal{F} .
$$

Then $\mathcal{F}$ is precompact in $C^{0}(B)$.

Let us choose $\beta$ as above and let us set $X=L^{\infty}$ and $B=Y=W^{-1, q}$, with $q<2$. We will apply the previous lemma to the sequence $\widetilde{\beta}\left(k^{\varepsilon}\right)$ with these spaces $X, B$ and $Y$. Obviously, $\widetilde{\beta}\left(k^{\varepsilon}\right)$ is uniformly bounded in $L^{\infty}(X)$. On 
the other hand, one has:

$$
\begin{aligned}
\partial_{t} \widetilde{\beta}\left(k^{\varepsilon}\right) & =-\mu_{\varepsilon}\left(k^{\varepsilon}\right) \beta^{\prime}\left(k^{\varepsilon}\right)\left|\nabla T_{M}\left(k^{\varepsilon}\right)\right|^{2}+\beta\left(k^{\varepsilon}\right) T_{\frac{1}{\varepsilon}}\left(\tau^{\varepsilon}: \nabla u^{\varepsilon}\right) \\
& +\nabla \cdot\left(\mu_{\varepsilon}\left(k^{\varepsilon}\right) \beta\left(k^{\varepsilon}\right) \nabla T_{M}\left(k^{\varepsilon}\right)+\beta\left(k^{\varepsilon}\right) B_{\varepsilon}\left(k^{\varepsilon}\right)-\widetilde{\beta}\left(k^{\varepsilon}\right) u^{\varepsilon}\right) \\
& -\beta^{\prime}\left(k^{\varepsilon}\right) \nabla T_{M}\left(k^{\varepsilon}\right) \cdot B_{\varepsilon}\left(k^{\varepsilon}\right) .
\end{aligned}
$$

Here, the first two terms in the right side converge strongly in $L^{1}(Q)$. The other terms are bounded in $L^{2}\left(H^{-1}\right)$. It is thus clear that, for some $\psi \in L^{1}(0, T)$ and some bounded set $\mathcal{B}$ in $L^{2}(0, T)$, one has (26). Consequently, passing again to a subsequence if necessary, $\widetilde{\beta}\left(k^{\varepsilon}\right)$ converges strongly in $C^{0}\left(W^{-1, q}\right)$, $\widetilde{\beta}(k) \in C^{0}\left(W^{-1, q}\right)$ and $\widetilde{\beta}(k)(0)=\widetilde{\beta}\left(k_{0}\right)$.

\section{The proof of theorem 2}

Let us assume that $B \equiv 0$ and $k_{0} \in L^{\infty}$ and repeat the proof of theorem 1 . Since (25) is satisfied for all $M>0$, we can also assume that $\nabla k^{\varepsilon} \rightarrow \nabla k$ a.e. in $Q$. Hence, for each $q<2$,

$$
\nabla k^{\varepsilon} \rightarrow \nabla k \quad \text { strongly in } L^{q}(Q) .
$$

Accordingly, $u^{\varepsilon} \cdot \nabla k^{\varepsilon}$ converges strongly, for instance, in $L^{1}(Q)$. Let us introduce the following functions:

$$
\widetilde{\mu}_{\varepsilon}(s)=\int_{0}^{s} \mu_{\varepsilon}(r) d r, \quad b_{\varepsilon}=\left(\widetilde{\mu}_{\varepsilon}\right)^{-1} .
$$

Let us set $v^{\varepsilon}=\widetilde{\mu}_{\varepsilon}\left(k^{\varepsilon}\right)$. We know that

$$
\partial_{t} b_{\varepsilon}\left(v^{\varepsilon}\right)-\Delta v^{\varepsilon}=F_{1}^{\varepsilon}+F_{2}^{\varepsilon} \text { in } Q,
$$

where $F_{1}^{\varepsilon}$ and $F_{2}^{\varepsilon}$ are given by (23). Let us see that $v^{\varepsilon}$ is bounded in $L^{q}\left(W_{0}^{1, q}\right)$ for each $q<2$. Since both $k^{\varepsilon}$ and $\nabla k^{\varepsilon}$ converge a.e., this will give (11) and (12).

¿From the results in [4] and [12], we know that it is sufficient to check the following:

$$
\iint_{Q}\left|\nabla T_{M}\left(v^{\varepsilon}\right)\right|^{2} \leq C M, \quad \frac{1}{m} \iint_{n \leq v^{\varepsilon} \leq n+m}\left|\nabla v^{\varepsilon}\right|^{2} \leq C(n, m) .
$$

First, by multiplying (27) by $T_{M}\left(v^{\varepsilon}\right)$ and integrating in time and space, the following inequality is easily obtained:

$$
\iint_{Q}\left|\nabla T_{M}\left(v^{\varepsilon}\right)\right|^{2} \leq M \iint_{Q}\left|F_{1}^{\varepsilon}+F_{2}^{\varepsilon}\right|+\int_{\Omega} Y_{M}^{\varepsilon}\left(v_{0}^{\varepsilon}\right) .
$$


Here,

$$
Y_{M}^{\varepsilon}(z)=\int_{0}^{z} b_{\varepsilon}^{\prime}(r) T_{M}(r) d r \quad \text { and } \quad v_{0}^{\varepsilon}=v^{\varepsilon}(0)=\widetilde{\mu}\left(k_{0}\right)
$$

(observe that $v_{0}^{\varepsilon}$ is bounded in $L^{\infty}$ ). The right side in (29) is $\leq C \dot{M}$, since $F_{1}^{\varepsilon}+F_{2}^{\varepsilon}$ converges strongly in $L^{1}(Q)$ and $0 \leq Y_{M}^{\varepsilon}(z) \leq \mu_{0} M z$ for all $z \geq 0$. Hence, the first part of (28) holds.

Now, let us use $\xi_{n, m}\left(v^{\varepsilon}\right)$ as test function in (27). This gives:

$$
\frac{1}{m} \iint_{n \leq v^{\varepsilon} \leq n+m}\left|\nabla v^{\varepsilon}\right|^{2} \leq \iint_{v^{\varepsilon} \geq n}\left|F_{1}^{\varepsilon}+F_{2}^{\varepsilon}\right|+\int_{\Omega} \widehat{\xi}_{n, m}^{\varepsilon}\left(v_{0}^{\varepsilon}\right) .
$$

Here, we have introduced the function $\widehat{\xi}_{n, m}$, given as follows:

$$
\widehat{\xi}_{n, m}^{\varepsilon}(z)=\int_{0}^{z} b_{\varepsilon}^{\prime}(r) \xi_{n, m}(r) d r \quad \forall z .
$$

It is clear that $0 \leq \widehat{\xi}_{n, m}^{\varepsilon}(z) \leq \mu_{0} z$ for all $z \geq 0$, whence we easily deduce the second estimate in $(28)$.

\section{$5 \quad$ The proof of theorem 3}

Let us set $u=u_{1}-u_{2}$ and $k=k_{1}-k_{2}$. It is not difficult to see (in the usual way) that

$$
\frac{1}{2} \frac{d}{d t}|u|^{2}+\nu\|u\|^{2} \leq-\left((u \cdot \nabla) u_{2}, u\right)-\left(k \Phi^{\prime}\left(\nabla u_{2}\right), \nabla u\right)
$$

in $(0, T)$. The first term in the right side can be bounded as follows:

$$
\left|\left((u \cdot \nabla) u_{2}, u\right)\right| \leq\left\|\nabla u_{2}\right\|_{L^{\infty}} \cdot|u|^{2} .
$$

The second one satisfies:

$$
\begin{aligned}
\left|\left(k \Phi^{\prime}\left(\nabla u_{2}\right), \nabla u\right)\right|= & \left|\left(\Phi^{\prime}\left(\nabla u_{2}\right) \nabla k, u\right)+\left(k \nabla \cdot\left(\Phi^{\prime}\left(\nabla u_{2}\right)\right), u\right)\right| \\
& \leq C\|k\| \cdot|u|+C\|k\|_{L^{b}}\left\|D^{2} u_{2}\right\|_{L^{r}} \cdot|u|,
\end{aligned}
$$

where $b$ is given by the identity $\frac{1}{b}+\frac{1}{r}+\frac{1}{2}=1$. Since $r>N$, one has $b<2^{*}$ and one obtains:

$$
\left|\left(k \Phi^{\prime}\left(\nabla u_{2}\right), \nabla u\right)\right| \leq C\left(1+\left\|D^{2} u_{2}\right\|_{L^{r}}\right)\|k\| \cdot|u| .
$$

Consequently, integrating (30) with respect to time in $(0, t)$, one is led to the following:

$$
\begin{aligned}
& \frac{1}{2}|u(t)|^{2}+\nu \int_{0}^{t}\|u(s)\|^{2} d s \leq \int_{0}^{t}\left\|\nabla u_{2}(s)\right\|_{L^{\infty}} \cdot|u(s)|^{2} d s \\
& \quad+\int_{0}^{t} C\left(1+\left\|D^{2} u_{2}(s)\right\|_{L^{r}}\right)\|k(s)\| \cdot|u(s)| d s .
\end{aligned}
$$


In the sequel, we will derive an estimate of $\|k\|$ in terms of $\|u\|$. In view of (31), it will then be possible to apply Gronwall's lemma and to deduce that $u \equiv 0$, i.e. $u_{1} \equiv u_{2}$.

First, notice that

$$
\left|\mu\left(k_{1}\right)-\mu\left(k_{2}\right)\right| \leq l|k|
$$

for some $l>0$. This happens because $\mu$ is locally Lipschitz-continuous and both $k_{1}$ and $k_{2}$ are bounded. In turn, this is a consequence of the fact that, in the PDE satisfied by $k_{i}$,

$$
\left|\nabla u_{i}\right|^{2} \in L^{\infty}(Q) \quad \text { and } \quad \Phi^{\prime}\left(\nabla u_{i}\right): \nabla u_{i} \in L^{\infty}(Q) .
$$

¿From the results in [10], taking into account that $k_{0} \in W^{2, \infty} \cap H_{0}^{1}$, one deduces:

$$
k_{i} \in L^{2}\left(W^{2, p}\right) \text { for all finite } p \quad \text { (in particular, } \nabla k_{i} \in L^{2}\left(L^{\infty}\right) \text { ). }
$$

¿From the equations satisfied by $k_{1}$ and $k_{2}$, we find:

$$
\begin{aligned}
\partial_{t} k & +u_{1} \cdot \nabla k-\nabla \cdot\left(\mu\left(k_{1}\right) \nabla k\right)=-u \cdot \nabla k_{2} \\
& +\nabla \cdot\left(\left(\mu\left(k_{1}\right)-\mu\left(k_{2}\right)\right) \nabla k_{2}\right)+\nu\left(\nabla u_{1}+\nabla u_{2}\right): \nabla u \\
& +k_{1}\left(\Phi^{\prime}\left(\nabla u_{1}\right): \nabla u_{1}-\Phi^{\prime}\left(\nabla u_{2}\right): \nabla u_{2}\right)+k \Phi^{\prime}\left(\nabla u_{2}\right): \nabla u_{2} .
\end{aligned}
$$

Hence,

$$
\begin{aligned}
& \frac{1}{2} \frac{d}{d t}|k|^{2}+\mu_{0}\|k\|^{2} \leq \int_{\Omega}\left|\nabla k_{2}\right| \cdot|k| \cdot|u| \\
& \quad+l \int_{\Omega}\left|\nabla k_{2}\right| \cdot|k| \cdot|\nabla k|+\nu \int_{\Omega}\left(\left|\nabla u_{1}\right|+\left|\nabla u_{2}\right|\right)|k| \cdot|\nabla u| \\
& \quad+C \int_{\Omega} k_{1}\left(1+\left|\nabla u_{2}\right|\right)|k| \cdot|\nabla u|+C \int_{\Omega}\left|\nabla u_{2}\right| \cdot|k|^{2} \\
& \quad \leq\left\|\nabla k_{2}\right\|_{L^{\infty}} \cdot|k| \cdot|u|+l\left\|\nabla k_{2}\right\|_{L^{\infty}} \cdot|k| \cdot\|k\| \\
& \quad+\left[\nu\left(\left\|\nabla u_{1}\right\|_{L^{\infty}}+\left\|\nabla u_{2}\right\|_{L^{\infty}}\right)+C\left\|k_{1}\right\|_{L^{\infty}}\left(1+\left\|\nabla u_{2}\right\|_{L^{\infty}}\right)\right]|k| \cdot\|u\| \\
& \quad+C\left\|\nabla u_{2}\right\|_{L^{\infty}} \cdot|k|^{2}
\end{aligned}
$$

in $(0, T)$. It is thus clear that, for some $g \in L^{1}(0, T)$, one can write:

$$
\frac{d}{d t}|k|^{2}+\mu_{0}\|k\|^{2} \leq g(t)|k|^{2}+\|u\|^{2} .
$$

After some elementary computations, we find

$$
\int_{0}^{t}\|k(s)\|^{2} d s \leq G \int_{0}^{t}\|u(s)\|^{2} d s \quad \text { for all } t,
$$


where $G$ is a constant only depending on $\|g\|_{L^{1}}$. Consequently, going back to (31), one deduces:

$$
\begin{aligned}
& |u(t)|^{2}+2 \nu \int_{0}^{t}\|u(s)\|^{2} d s \leq 2 \int_{0}^{t}\left\|\nabla u_{2}(s)\right\|_{L^{\infty}} \cdot|u(s)|^{2} d s \\
& +C\left(\int_{0}^{t}\|u(s)\|^{2} d s\right)^{\frac{1}{2}}\left(\int_{0}^{t}\left(1+\left\|D^{2} u_{2}(s)\right\|_{L^{r}}\right)^{2}|u(s)|^{2} d s\right)^{\frac{1}{2}} \\
& \leq \nu \int_{0}^{t}\|u(s)\|^{2} d s+C \int_{0}^{t}\left(1+\left\|\nabla u_{2}(s)\right\|_{L^{\infty}}+\left\|D^{2} u_{2}(s)\right\|_{L^{r}}^{2}\right)|u(s)|^{2} d s .
\end{aligned}
$$

In other words, we have proved that

$$
|u(t)|^{2}+\nu \int_{0}^{t}\|u(s)\|^{2} d s \leq \int_{0}^{t} h(s)|u(s)|^{2} d s \quad \text { for all } t
$$

for some nonnegative $h \in L^{1}(0, T)$. This implies $u \equiv 0$; from (32), one also has $k \equiv 0$. Therefore, the proof is completed.

Acknowledgment: The authors are indebted to D. Blanchard, for several fruitful discussions.

\section{References}

[1] J. BARAnger, A. Mikelić - Stationary solutions to a quasi-Newtonian flow with viscous heating, Math. Models and Methods in Appl. Sciences, Vol. 5, No. 6 (1995), 725-738.

[2] P. Benilan, L. Boccardo, T. Gallouët, R. Gariepy, J.L. Vázquez - On the $p$-Laplacian on $L^{1}$, to appear.

[3] D. Blanchard, H. RedWAne - Renormalized solutions for a class of nonlinear evolution problems, to appear in Journal Math. Pures Appl.; see also C. R. Acad. Sci. Paris, t. 319, Série I, p. 831-835, 1994.

[4] L. Bocchrdo, T. GAllouËT - Nonlinear elliptic and parabolic equations involving measure data, J. Funct. Anal. 87, 1989, p. 149-169.

[5] L. Boccardo, J.I. Díaz, D. Giachetti, F. Murat - Existence of a solution for a weaker form of a nonlinear elliptic equation, In "Recent Advances in Nonlinear Elliptic and Parabolic Problems", P. Benilan et al. eds., Pitman Research Notes in Math., 208, Longman, Harlow 1989.

[6] P. Bradshaw - The understanding and prediction of turbulent flow, Aeronautical J., july 1972, p. 403-418. 
[7] B. Climent - Thesis, University of Sevilla (Spain), 1996.

[8] B. Climent, E. Fernández-CARA - Existence and uniqueness results for a coupled problem related to the stationary Navier-Stokes system, to appear in Journal Math. Pures Appl.

[9] R. DiPerna, P.L. Lions - On the Cauchy problem for Boltzmann equations: global existence and weak stability, Annals of Math. (2) 130 (1989), No. 2, p. 321-366.

[10] O.A. Ladyzhenskaya, V.A. Solonnikov, N.N. Ural'CeVa -Linear and quasi-linear equations of parabolic type, Trans. Math. Monographs, Vol. 23, A.M.S., Providence 1968.

[11] R. Lewandowski - Les équations de Stokes et de Navier-Stokes couplées avec l'équation de l'énergie cinétique turbulente, C. R. Acad. Sci. Paris, t. 381, Série I, p. 1097-1102, 1994.

[12] P.L. Lions, F. Murat - Solutions renormalisées d'équations elliptiques, to appear.

[13] F. MURAT - Soluciones renormalizadas de EDP elípticas no lineales, Research Report R93023, LAN, University Paris VI, 1993.

[14] M. Nallasamy - Turbulence models and their applications to the prediction of turbulent flows, Computers \& Fluids, Vol. 15, No. 2, pp. 151-194, 1987.

[15] W.C. Reynolds - Computation of turbulent flows, Annual Reviews (1976), p. 183-207.

[16] J. Simon - Compact sets in the space $L^{p}(0, T ; B)$, Annali Mat. Pura Appl. (IV), Vol CXLVI (1987), p. 65-96.

[17] J. Simon - Existencia de solución del problema de Navier-Stokes con densidad variable, Lectures at the University of Sevilla (Spain), 1990. 